\title{
Shape Optimization with the Level-Set-Method Using Local Volume Constraints
}

\author{
Simon H. Hesse · Lukas F. Leidinger · Johannes Kremheller · Dirk Lukaszewicz · \\ Fabian Duddeck
}

Received: May 8, 2017 / Accepted: t.b.d.

\begin{abstract}
This paper presents a method to locally constrain multiple material volume domains for structural optimization with the Level Set Method (LSM). Two different Lagrangian formulations and multiplier update methods are used, for both the global and local problem. The local volume domains can be constrained by both equality and inequality constraints. The optimization objective is compliance minimization for well-posed statically loaded structures. For validation, several example problems are established and solved using the proposed method. Results show that the volume ratios for user established sub-domains can be controlled successfully. The local constraint values are met accurately in the case of equality constraints and remain in their feasible domain in the case of inequality constraints. Optimization results are not significantly hindered by the introduction of local volume constraints for comparable problems.
\end{abstract}

Keywords Level Set Method · Shape Optimization · Local Constraints · Local Volume

\section{S.H. Hesse}

BMW Group, Research and Innovation Centre

Knorrstrasse 147

D-80788, Munich, Germany

E-mail: simon.hesse@hesseng.nl

L.F. Leidinger · J. Kremheller · F. Duddeck

Computational Mechanics

Technical University of Munich (TUM)

Arcisstrasse 21

D-80333, Munich, Germany

D.H.-J.A. Lukaszewicz

BMW Group, Research and Innovation Centre

Knorrstrasse 147

D-80788, Munich, Germany

\section{Introduction}

The developments in the field of structural optimization have been numerous and successful over the past decades. Within this field a rudimentary classification is made between size, shape and topology optimization [19].

The popular choice is the field of topology optimization. Especially density based methods, like Solid Isotropic Material with Penalization (SIMP) $[6,35]$, gained broad interest. However, recently the Level Set Method (LSM) for structural optimization is gaining attention as well. The LSM originated from research by Osher and Sethian [23]. They developed the method as a means to follow fronts propagating with curvature-dependent speed. Their new scheme allowed for following the motion of an $N-1$ dimensional surface in $N$ space dimensions. The front of this surface could be advected over time by solving the so-called HamiltonJacobi Partial Differential Equation (PDE), also called the Level Set Equation (LSE), with an appropriate normal velocity that is the moving boundary velocity normal to the interface. They identified that topological merging and breaking was managed naturally by their method. The level-set method is versatile and therefore found a broad application in different areas, such as fluid mechanics, phase transitions, image processing and solid modeling in CAD.

Sethian and Wiegmann [28] built on the topological versatility of the LSM and were first to develop a structural optimization method using this method. The structural rigidity was improved by using ad-hoc criteria based on the vonMises equivalent stress.

Osher and Santosa [24] first introduced the use of shape sensitivities with the LSM for structural optimization. The elasticity tensor remained constant over the entire domain. The shape sensitivity was used as the normal velocity of the boundary and the Level Set Function (LSF) was updated, 
thus moving the boundary by solving the LSE with an upwind numerical Finite Difference (FD) scheme.

Both Allaire et al. [3,4] and Wang et al. [30] independently developed a generalized structural optimization method based on the level-set framework and classical shape sensitivity analysis. Their method is considered a combination of the before mentioned developments and made the LSM applicable to a wider range of structural optimization problems. They both solved a selection of typical optimization problems, for example compliance minimization, proving the potential of the LSM for structural optimization.

The LSMs introduced above, also known as the Conventionial LSM, rely on solving the Hamilton-Jacobi equation to advance the level set boundary. Computationally expensive numerical techniques, such as the upwind scheme, are necessary to accomplish this task.

The issue of solving the Hamilton-Jacobi equation can be circumvented by using a so-called parameterized LSM $[10,9,25,31,22]$. The difference with the common LSM is that with the parameterized approach, the geometry description is entirely decoupled from the finite element discretization. Parameterization of the LSF allows the HamiltonJacobi equation to be discretized into a system of mathematically more convenient coupled Ordinary Differential Equations (ODEs). This means the sensitivities can be determined explicitly and a larger variety of optimization algorithms can be applied, e.g. stochastic methods such as genetic algorithms or mathematical programming such as Method of Moving Asymptotes (MMA). The parameterized approach to the level set based topology optimization shows promising results. However, the type of parameterization and the number of parameters influence the maximum level of detail of the material boundary that can be represented.

In case conventional LSMs are used, the choice of optimization algorithms is limited. Often a variant of the indirect search method with the steepest-descent approach based on the Optimality Criteria (OC) is used $[4,30,32]$. OC are the conditions a function must satisfy at its minimum point [5] (e.g. Karush-Kuhn-Tucker (KKT) conditions). Algorithms that try to find solutions to these conditions are often called OC or indirect methods. Another approach is the direct search method, which starts with an estimate and searches the design space for optimum points, iteratively improving the design until the OC are satisfied [5].

Direct search methods, such as Sequential Quadratic Programming (SQP) and MMA, work well when the response functions are an explicit function of the design variables, such as the element densities in the SIMP method or the parameters in the parameterized LSM approach. However, in conventional LSM, the behavior of the response functions as a function of the level set variables is often very different. The reason is the non-linear relation between the LSF and the material domain [12]. The Hamilton-Jacobi
PDE or LSE are both space and time dependent, with a velocity component driving the evolution of the equation. By introducing a pseudo-time component, the normal velocity of the level set boundary can be related to the shape derivative of the material domain. However, this relation is implicit in nature and leaves indirect search methods as the favorable method for conventional LSMs.

A significant downside of the indirect OC method is the difficulty to include multiple design constraints. The sensitivities collected in the sensitivity field over the design domain can be used directly to construct the Hamilton-Jacobi velocity field. If design constraints are introduced, the constrained optimization problem needs to be transformed into an unconstrained one. The popular choices are penalty or augmented Lagrange multiplier formulations. Classically, these methods have difficulty handling a large number of design constraints.

The penalty method was implemented by Allaire et al. $[4,1]$. They referred to the penalty factor as a fixed Lagrange multiplier, however, the method is unable to enforce the constraints exactly and therefore requires trial and error to set the desired penalty factor. The augmented Lagrange multiplier method is considerably more popular, in large due to its ability to converge to good optima while matching constraints values exactly. Furthermore, it can be proven that the augmented Lagrange method converges faster than commonly used penalty methods [5] and it is well-known for its numerical stability. Different variants of the method were used by Challis [8], Luo et al. [21], van Dijk et al. [12] and Otomori et al. [26].

Although the LSM has gained a broader interest over the past decade, relatively little research was done on including multiple constraint, especially when it concerns the conventional LSM. The majority of the multi-constrained optimization problems in topology optimization are concerned with strength or stress constraints. The strength is maximized by limiting local stresses. Examples can be found that use the density based SIMP method to solve a structural optimization problem with local stress constraints [15,34,27,20,7]. With the LSM however, the research is limited and involves a different variant of the problem. In many cases a stressminimization problem is solved to achieve a similar effect; mitigating the introduction of many stress-based design constraints by reformulating the objective function and deriving the new sensitivities $[2,29,17]$.

Structural optimization methods are used extensively in automotive engineering; for reducing weight and optimizing crash performance, among others [14]. During a crash, the vehicle has to absorb a significant amount of the impact energy in order to protect the occupants. The impact force during a crash determines the deceleration of the car, which, when transferred to the occupants, is an important injury criterion. Advanced composite materials, such as Carbon Fiber 
Reinforced Plastic (CFRP), are now used to decrease vehicle weight without losing stiffness and strength. Ideally these materials are also incorporated in the crash energy absorbing vehicle areas. However, it is challenging to develop these structures such that they are low weight, provide stiffness and have a favorable deceleration over time, also called the crash pulse.

One of the interesting aspects of CFRP composites is that its crushing force may be linear proportional to the amount of material in the impacted area [16]. Therefore controlling the distribution of the material while optimizing the overall stiffness might benefit the crash worthiness. This could be achieved by controlling the volume in multiple local domains in the total design domain. However, no method allows for large control over local volume constraints. Especially the control over local volume, might help the early phase design of high performance CFRP crash absorbing structures.

The topic of multiple volume constraints belongs to the aforementioned research into multi-constrained design problem, although little on this topic is found in literature. Especially the control over local volume, might help the early design phase of high performance CFRP crash absorbing structures. In this paper, we present a level-set based, robust structural optimization framework, which can handle multiple, arbitrarily placed, volume constraints. The volume constraints are applied to non-overlapping sub-domains of the design domain.

The method introduced in this paper will be applied to two validation examples; the well known Mitchel bridge and an example from the automotive industry. The automotive example will show how the method can be applied to find optimal load paths for an idealized CFRP structure under impact. The method of local volume constraints is used to enforce a constant material volume in the direction of impact.

This paper is structured as follows. In Section 2, the LSM formulations and procedures are explained. The compliance optimization problem is explained in Section 3 and the optimization methods that were used are presented in Section 4 . The validation examples are explained and the results discussed in Section 5 , followed by a critical reflection in Section 6. Finally the conclusions are presented in Section 7.

\section{Level Set Method}

In this section the principles behind the Level Set Method (LSM) are explained in the context of our research. For a thorough discussion on the method, the reader is referred to the works by $[4,30,13]$.

Let $D$ be a bounded reference domain and $\Omega$ a domain filled with solid material within $D$, such that $\Omega \subset D$. The complementary domain representing a void that exists, i.e. a void domain is then expressed as $D \backslash \Omega$. In the Level Set framework, the material domain or design boundary $\partial \Omega$ is embedded implicitly as the zero Level Set of a onedimensional-higher LSF $\phi(\mathbf{x})$, where $\mathbf{x}$ stands for a position within $D$. Each part in the design domain is then defined as follows:

$$
\begin{aligned}
& \phi(\mathbf{x})<0 \forall \mathbf{x} \in \Omega \backslash(\partial \Omega \backslash \partial D) \\
& \phi(\mathbf{x})=0 \forall \mathbf{x} \in \partial \Omega \backslash \partial D \\
& \phi(\mathbf{x})>0 \forall \mathbf{x} \in D \backslash \Omega
\end{aligned}
$$

where $\partial D$ is the boundary of the reference domain $D$. With the LSF as presented in equation (1), any shape or topology of the material domain $\Omega$ can be represented within the reference domain $D$. In the definition from equation (1); $\phi>0$, represents a void domain and $\phi<0$ represents a material domain. This relation is depicted in Figures 1(a) and 1(b).

Now we introduce a fictitious time $t$, and assume that the LSF is also implicitly a function of $t$, to represent shape and topology changes in the material domain $\Omega$ over time. That is, as time $t$ is advanced, the boundary $\partial \Omega$ on $\phi(\mathbf{x})=0$ is updated as an evolving boundary process that reaches an optimal configuration. In the LSM framework, the moving boundary can be tracked by solving the so-called HamiltonJacobi equation or LSE:

$\frac{\partial \phi(\mathbf{x}, t)}{\partial t}+\nabla \phi(\mathbf{x}, t) \frac{\mathrm{d} \mathbf{x}}{\mathrm{d} t}=0$

where $\phi(\mathbf{x}, t)$ is the time dependent LSF. The boundary normal velocity $V_{N}(\mathbf{x}, t)$ of the material boundary can be derived as follows:

$V_{N}(\mathbf{x}, t)=\mathbf{V}(\mathbf{x}, t) \frac{\nabla \phi(\mathbf{x}, t)}{|\nabla \phi(\mathbf{x}, t)|}$

with: $\quad \mathbf{V}(\mathbf{x}, t)=\frac{\mathrm{d} \mathbf{x}}{\mathrm{d} t}$

The normal velocity $V_{N}$ from equation (3) is substituted into the LSE from equation (2) to produce:

$$
\frac{\partial \phi(\mathbf{x}, t)}{\partial t}+V_{N}(\mathbf{x}, t)|\nabla \phi(\mathbf{x}, t)|=0
$$

The normal velocity is related to the sensitivity of the objective function with respect to the boundary variation. Hence, moving the material boundary along the normal direction is equivalent to updating the $\operatorname{LSF} \phi(\mathbf{x})$ by solving the LSE. Thus, the structural optimization problem can be solved by providing appropriate velocity function values $V_{N}(\mathbf{x}, t)$ for use in equation (4). 


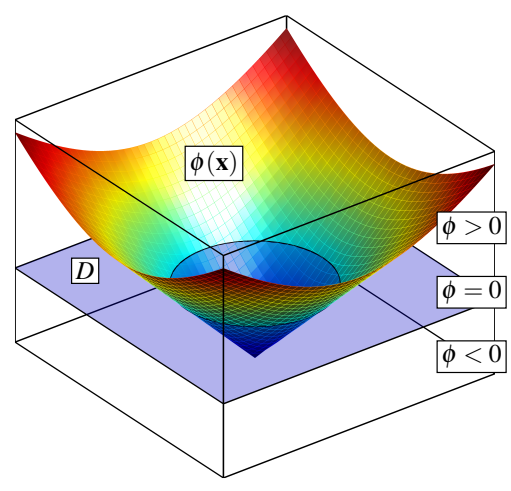

(a) A LSF with a level-set representing the material domain $D$.

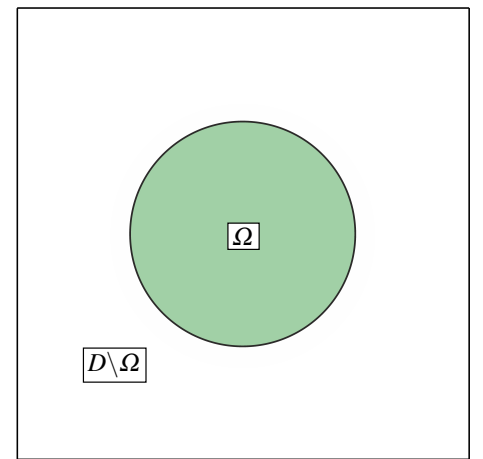

(b) The geometry boundary in the $2 \mathrm{D}$ Level Set domain corresponding to the LSF.

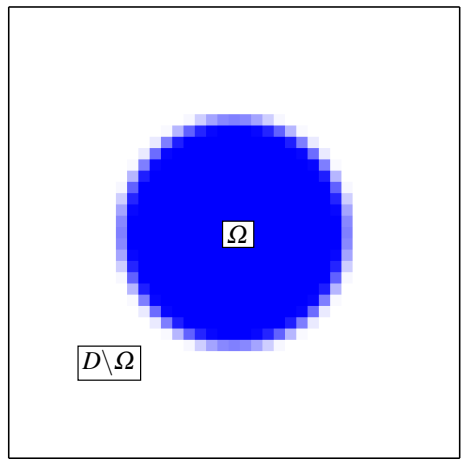

(c) The FE-representation after densitybased mapping.

Fig. 1 Example of the material domain description with the LSM (figures a and b) and the FE description of the material domain (figure c).

\subsection{Geometry Mapping}

In this paper the geometry boundary is described by an intermediate density field, indicating the amount of material at each point of the design domain. The function used to determine the material density should be differentiable in order to derive the shape sensitivities consistently. The Heaviside function provides that capability and is therefore often used in the LSM. The Heaviside function can be used to determine the material density $\rho(\phi)$ as follows:

$\rho(\phi)=H(\phi(\mathbf{x}))$

However, the exact definition of the Heaviside function is discontinuous and can lead to numerical issues during optimization. The binary definition of the material density reduces the accuracy of the LSF update procedure. In order to solve this problem, a relaxed Heaviside function definition, based on the work by [30], is used. The relaxed Heaviside and the derivative thereof are found in equations (6) and (7).

$\tilde{H}(\phi(\mathbf{x}))= \begin{cases}1 & \forall \phi<-h \\ \frac{1}{4}\left(\frac{\phi}{h}\right)^{3}-\frac{3}{4}\left(\frac{\phi}{h}\right)+\frac{1}{2} & \forall-h \leq \phi \leq h \\ 0 & \forall \phi>h\end{cases}$

The derivative of $\tilde{H}(\phi(\mathbf{x}))$ in equation (6) is the relaxed Dirac delta function $\tilde{\delta}(\phi(\mathbf{x}))$ :

$\tilde{\delta}(\phi(\mathbf{x}))= \begin{cases}\frac{3}{4}\left(\frac{\phi^{2}}{h^{3}}\right)-\frac{3}{4 h} & \forall-h \leq \phi \leq h \\ 0 & \forall \phi<-h, \quad \phi>h\end{cases}$

The definition in equation (5) can result in a zero density. This might lead to singularity in solving the mechanical problem during the FE-analysis. To resolve this issue the definition in equation (5) is often rewritten as follows:

$\rho(\phi)=\varepsilon+(1-\varepsilon) H(\phi(\mathbf{x}))$

where $\varepsilon$ is a small number representing a minimum density. In the research presented in this paper, the elements with zero density are deleted. Therefore no singularities can arise and thus no minimal material density is required.

Calculating the volume fraction The LSF is discretized on the FE-grid with the LSF values on the nodes. Consequently the relaxed Heaviside values, $\tilde{H}(\phi(\mathbf{x}))$, are also given on the nodes. However, for the element volume fraction we need to calculate $\rho(\phi)$ on an element level. This means it is necessary to calculate $\tilde{H}(\phi(\mathbf{x}))$ on element level. This necessity is unfortunately often left out in literature and left up to the user to discover / realize, although van Dijk et al. [12] mention this issue in their paper. The common way of doing this is to average the nodal Heaviside values to the element level. However, while $\phi(\mathbf{x})$ might be linearly interpolated on the element with use of the element shape functions $\mathbf{N}$, the Heaviside function, $\tilde{H}(\phi(\mathbf{x}))$, cannot. The relation between $\tilde{H}$ and $\phi$ is not linear. Depending on the bandwidth, $h$, large areas of the element might have a constant value for $\tilde{H}$ of 1 or 0 . Simply taking the average can lead to an unchanging element density, while the change in nodal LSF values, $\phi$, should suggest a change in density. To ensure a consistent sensitivity calculation, the element volume fraction should be calculated by integrating $\tilde{H}(\phi(\mathbf{x}))$ over the element and divide by the total element volume:

$\left.\tilde{H}(\phi(\mathbf{x}))\right|_{e}=\frac{\int_{D_{e}} \tilde{H}(\phi(\mathbf{x})) \mathrm{d} D_{e}}{\int_{D_{e}} \mathrm{~d} D_{e}}$

This is unfortunately a computationally intensive procedure and slows the optimization process considerably. The averaging method will provide results with a relatively small error compared to the integration method when the bandwidth, $h$, is sufficiently large, or at least larger than the critical element side length. The averaging method will work with lower $h$-values, but this will result in inconsistent derivation of the sensitivities. 


\subsection{Update Procedure}

The LSF is updated by providing appropriate values for $V_{N}$ and numerically solving the LSE in equation (4). Different numerical techniques for solving this PDE are currently being researched, popular and commonly used is the upwindscheme $[4,30,8]$. This scheme solves the LSE from equation (4) both temporally and spatially. In this paper we use the method from Yamasaki et al. [32] to update the LSE. By ensuring the Signed Distance Function (SDF) property of the LSF at all times, the LSE from equation (4) can be rewritten as follows:

$\frac{\partial \phi(\mathbf{x}, t)}{\partial t}+V_{N}(\mathbf{x}, t)=0$

with the SDF property as:

$\|\nabla \phi(\mathbf{x})\|=1 \quad \forall \quad \mathbf{x} \in D$

The geometric reinitialization method from [32] is applied to ensure the SDF property is kept at all times. However, this reinitialization technique is computationally expensive; every grid point is compared to all points on the Level Set boundary. Only LSF values in the vicinity of the material boundary described by $\phi=0$ are updated, because of the Dirac delta function within the shape sensitivity. Here the value for bandwidth $h$ determines the area of influence around the boundary. Knowing this, the computational effort required for the reinitialization method can be significantly reduced. Instead of comparing all grid points, only the grid points located within the bandwidth $h$ of the material boundary $\phi=0$ are reinitialized.

Now the LSF is updated explicitly as follows:

$$
\begin{aligned}
& \frac{\phi(\mathbf{x}, t+\Delta t)-\phi(\mathbf{x}, t)}{\Delta t}+V_{N}(\mathbf{x}, t)=0 \\
& \phi(\mathbf{x}, t+\Delta t)=-V_{N}(\mathbf{x}, t) \Delta t+\phi(\mathbf{x}, t)
\end{aligned}
$$

with a maximum time step defined by the CourantFriedrichs-Lewy (CFL) condition:

$\Delta t_{\text {crit }} \leq \frac{l_{\text {crit }}}{\max \left(V_{N}\right)}$

where $l_{\text {crit }}$ is the critical element length, e.g. the minimal element side length in the design domain. To further increase the stability of the overall optimization, $\Delta t_{\text {crit }}$ is multiplied with a positive factor, $t_{\text {fac }} \leq 1$. Lower $t_{\text {fac }} \leq 1$ increases the stability, but at the cost of optimization speed.

\subsection{Regularization}

Regularization is often employed during the update procedure of the LSF to remove numerical artifacts, simplify the problem, improve the convergence behavior and avoid convergence to local minima with poor performance [13].

Two types of regularization are applied in the presented LSM approach. The first regularization is designed to smooth the sensitivities before updating the LSE and is based on the Helmholtz-type PDE [33,18]. The second type adds a penalty function based on the zero level-set perimeter.

Helmholtz-type regularization Regularization is based here on the Helmholtz equation functions as a convolution filter. In other words, it diffuses or smooths the velocity field on and around the zero level-set interface. The Helmholtz derived equation used in this research is taken from the work by Yamasaki et al. [33]:

$$
(\mathbf{L}+\mathbf{H}) \tilde{\mathbf{V}}_{N}=\mathbf{L} \mathbf{V}_{N}
$$

where $\mathbf{V}_{N}$ is the nodal value vector of boundary normal velocities, i.e. the shape sensitivities, and $\tilde{\mathbf{V}}_{N}$ is the nodal value vector of the smoothed velocities. The matrices $\mathbf{L}$ and $\mathbf{H}$ are described as follows:

$$
\begin{aligned}
& \mathbf{L}=\bigcup_{e=1}^{N_{e}} \int_{D_{e}} \mathbf{N N}^{\mathrm{T}} \mathrm{d} D_{e} \\
& \mathbf{H}=\bigcup_{e=1}^{N_{e}} \int_{D_{e}} R^{2}\left(\mathbf{N}_{x} \mathbf{N}_{x}^{\mathrm{T}}+\mathbf{N}_{y} \mathbf{N}_{y}^{\mathrm{T}}\right) \mathrm{d} D_{e}
\end{aligned}
$$

with: $\quad \mathbf{N}_{x}=\frac{\partial \mathbf{N}}{\partial \mathbf{x}}, \quad \mathbf{N}_{y}=\frac{\partial \mathbf{N}}{\partial \mathbf{y}}$

where $N_{e}$ is the total number of elements in the FEdiscretization, $D_{e}$ the element domain and $R$ the regularization coefficient. For $R=0$ no smoothing takes place and $\tilde{\mathbf{V}}_{N}=\mathbf{V}_{N}$, for $R \rightarrow \infty$ the equation (14) becomes the Poisson's equation, i.e. the velocities are perfectly smoothed and equal. Equation (14) can be easily solved using the Finite Element Method (FEM), taking care that a four point Gaussian integration scheme is used. The smoothing parameter, $R$, is normalized with respect to the critical element length $l_{\text {crit }} ; \tilde{R}=\frac{R}{l_{\text {crit }}}$

Parameter penalty regularization The perimeter based regularization is based on penalizing shape updates that increase the total perimeter of the geometry. It is a common method of regularization and found for example in the works by Allaire et al. and Luo et al. [4,21]. The perimeter is defined as:

$$
P(\Omega)=\int_{\partial \Omega} \mathrm{d} S
$$

The perimeter $P(\Omega)$ is constrained by including it in the Lagrange formulation of the optimization problem as a penalty constraint. The shape sensitivity of the perimeter is equal to 
the mean curvature of the boundary, which is equal to the divergence of the boundary normal:

$\kappa=\operatorname{div}\left(\frac{\nabla \phi(\mathbf{x})}{|\nabla \phi(\mathbf{x})|}\right)$

The total shape sensitivity is then formed by extending the current velocity field with the the mean curvature field, $\kappa$, multiplied by a certain penalty, $\beta$ :

$V_{N}^{\text {new }}=V_{N}^{\text {old }}+\beta \kappa$

\section{Optimization Problem}

In this section the optimization problem will be discussed and the multi-domain volume constraints introduced. The objective is to minimize the compliance of a design subject to both the global and local volume constraint(s).

\subsection{Single or Global Volume Constraint}

The optimization problem of minimizing the structural compliance subject to a single volume constraint is defined as follows:

$$
\begin{aligned}
& \text { Minimize: } c(\phi(\mathbf{x})) \\
& \text { Subject to: } g(\phi(\mathbf{x}))=V(\phi(\mathbf{x}))-V_{\max } \leq 0 \\
& \tilde{\mathbf{K}} \cdot \mathbf{u}-\mathbf{f}^{\mathrm{ext}}=\mathbf{0} \\
& \phi \in \Psi
\end{aligned}
$$

where $c$ is the compliance, $V$ equals the normalized volume fraction of the material domain $\Omega$ on $D$ and $V_{\max }$ equals the maximum allowed normalized volume fraction of $\Omega$ on the design domain $D$. The global stiffness matrix is denoted by $\tilde{\mathbf{K}}, \mathbf{u}$ is the nodal displacement vector and $\Psi$ is the space of allowable LSF values $\phi$. The structural problem is only subjected to traction forces $\mathbf{f}^{\text {ext }}$ and is not subjected to body forces. The Lagrangian $\mathscr{L}$ is derived in order to transform the inequality constrained optimization problem into an unconstrained problem:

$\mathscr{L}(\phi(\mathbf{x}))=c(\phi(\mathbf{x}))+\lambda(g(\phi(\mathbf{x})))$

where the compliance $c$ and constraint $g$ are taken as:

$c=\sum_{e=1}^{N_{e}} \tilde{H}(\phi(\mathbf{x})) \mathbf{u}_{e}^{T} \cdot \mathbf{K}_{e} \cdot \mathbf{u}_{e}$

$g=\sum_{e=1}^{N_{e}} \tilde{H}(\phi(\mathbf{x}))$
Based on the discussion in [30], the normal velocity $V_{N}$ from the LSE is expressed as follows:

$V_{N}(\mathbf{x}, t)=\frac{\mathrm{d} \mathscr{L}}{\mathrm{d} \phi}$

The shape derivative of the Lagrangian is defined as follows:

$\frac{\mathrm{d} \mathscr{L}}{\mathrm{d} \phi}=\sum_{e=1}^{N_{e}} \tilde{\delta}(\phi(\mathbf{x}))\left[-\mathbf{u}_{e}^{T} \cdot \mathbf{K}_{e} \cdot \mathbf{u}_{e}+\lambda\right]$

where $N_{e}$ is the total number of elements, $\mathbf{K}_{e}$ is the element stiffness matrix. The shape derivatives of the objective and constraint are derived in detail in Appendix A.

\subsection{Multi-domain Local Volume Constraints}

We introduce a method to constrain multiple local volume domains. With this method the user can assert more control on the topological development during optimization. By restraining the volume in sub-domains, the optimization algorithm is forced to find different solutions. This extended control can be used to create optimal topology, which better fit the intended application of the user.

Let us define three possible domain descriptions:

1. $D_{\text {glob }}$ : This is the domain that is left after all local domains are established.

2. $D_{\text {loc, eq }}$ : This is the combined domain of all local volume domains, $D_{j}^{\text {loc, eq }}$, that are controlled via equality volume fraction constraints.

3. $D_{\text {loc, ineq }}$ This is the combined domain of all local volume domains, $D_{l}^{\text {loc, ineq }}$, that are controlled via inequality volume fraction constraints.

The volume fraction of the entire design domain is still controlled by the previously introduced Lagrange multiplier $\lambda$, which will now be denoted $\lambda^{\text {glob }}$. Extra multipliers $\lambda_{i}^{\text {loc }}$ for $i=1 \ldots N_{\text {dom }}$ are introduced for $N_{\text {dom volume domains. The }}$ local volume domains are controlled in much the same way as the global volume constraint; the multiplier belonging to a volume domain is updated with the same update method as the global multiplier. The difference is that the update is based on the difference between the local volume fraction $V_{i}^{\text {loc }}$ and the local required Volume fraction $V_{i}^{\text {loc, req }}$ or allowed maximum volume fraction $V_{i}^{\mathrm{loc}, \max }$ for $i=1 \ldots N_{\mathrm{dom}}$.

Another important difference with the global constraint occurs for local inequality constraints. The local equality constraint is handled much the same way as the global constraint, as the same physical relation between volume fraction and compliance is true. The equality and inequality constraints are defined as follows:

$h_{j}(\phi(\mathbf{x}))=V_{j}^{\mathrm{loc}}-V_{j}^{\mathrm{loc}, \text { req }}=0$
$g_{l}(\phi(\mathbf{x}))=V_{l}^{\mathrm{loc}}-V_{l}^{\mathrm{loc}, \max } \leq 0$ 
However, contrary to the global inequality constraint, which behaves like an equality constraint, local inequality volume constraints are now physically sensible. This is due to the fact that each local volume domain also contributes to the global volume fraction, which is constrained as well. It is therefore possible that a local domain with low strain energy reduces in volume in favor of an area where the strain energy is higher, as long as the global volume constraint is met. This could result in local volume domains having a lower than maximum volume fraction at optimum. This behavior might be preferable and therefore a method is introduced to allow for local inequality volume constraints. The method works by comparing the local multiplier with the global one and taking the maximum, which allows to drive the local volume fraction further down if required.

The Lagrangian formulation of the optimization problem now is described as follows:

$$
\begin{aligned}
\mathscr{L}(\phi(\mathbf{x}))= & c(\phi(\mathbf{x}))+\lambda^{\text {glob }} g(\phi(\mathbf{x})) \\
& \ldots+\sum_{j=1}^{N_{\text {dom }}^{e q}} \lambda_{j}^{\text {loc }} h_{j}(\phi(\mathbf{x})) \\
& \ldots+\sum_{l=1}^{N_{\text {dom }}^{\text {ineq }}} \max \left[\lambda_{l}^{\text {loc }}, \lambda^{\text {glob }}\right] g_{l}(\phi(\mathbf{x}))
\end{aligned}
$$

where $\lambda_{j}^{\text {loc }}$ and $\lambda_{l}^{\text {loc }}$ are the local Lagrange multipliers for $N_{\text {dom }}^{e q}$ equality and $N_{\text {dom }}^{\text {ineq }}$ inequality constrained local volume domains. This optimization problem can be interpreted as finding a configuration, which exhibits a stationary Lagrangian $\mathscr{L}(\phi(\mathbf{x}))$ with respect to shape $\Omega$ and Lagrange multipliers $\lambda^{\text {glob }}, \lambda_{j}^{\text {loc }}$ and $\lambda_{l}^{\text {loc }}$. In order to find such a configuration, the KKT optimality conditions must hold. The boundary normal velocity $V_{N}$ can now be derived as:

$$
V_{N}=\tilde{\delta}(\phi(\mathbf{x}))\left[-\mathbf{u}^{\mathrm{T}} \mathbf{K u}+\Lambda\right]
$$

with: $\Lambda= \begin{cases}\lambda_{\text {glob }} & \forall \mathbf{x} \in D_{\text {glob }} \\ \lambda^{\text {loc, eq }} & \forall \mathbf{x} \in D_{\text {loc, eq }} \\ \max \left(\lambda^{\text {glob }}, \lambda^{\text {loc }, \text { ineq }}\right) & \forall \mathbf{x} \in D_{\text {loc, ineq }}\end{cases}$

Equation (30) shows three distinct cases.

- $D_{\text {glob }}$ : Only the multiplier $\lambda^{\text {glob }}$ is applied in this domain.

- $D_{\text {loc, eq }}$ : Only the multipliers $\lambda_{j}^{\text {loc }}$ are applied in these sub-domains. The volume of sub-domain $j$ at optimum will be equal to $V_{j}^{\text {loc,req }}$.

- $D_{\text {loc, ineq }}$ : Both the multipliers $\lambda_{l}^{\text {loc }}$ and $\lambda^{\text {glob }}$ are applied in these sub-domains. The volume of sub-domain $l$ at optimum will be equal to or lower than $V_{l}^{\text {loc,max }}$. The inequality is handled by the interaction between both multipliers.

The presented method for introducing multiple local volume constraints can be applied to arbitrary non-overlapping local domains. Care has to be taken that feasible volume requirements are chosen. A material distribution should exist that fulfills all constraint requirements without mechanical singularities.

\section{Optimization Methods or Numerical Implementation}

In this section the numerical framework to solve the optimization problem is explained. A normalization parameter $C$ is used, based on research by Otomori et al. [26], which normalizes the sensitivities, such that it makes the proposed LSM framework largely problem independent:

$V_{N}=\tilde{\delta}(\phi(\mathbf{x}))\left[-C\left(\mathbf{u}^{\mathrm{T}} \mathbf{K} \mathbf{u}\right)+\Lambda\right]$

The normalization parameter is defined as:

$C=\frac{N_{n}}{\sum_{e=1}^{N_{e}} \rho_{e} \mathbf{u}_{e}^{\mathrm{T}} \mathbf{K}_{\mathbf{e}} \mathbf{u}_{e}}$

where $N_{n}$ is the number of nodes in the LSF discretization and $N_{e}$ the number of elements.

The sub-domain volumes are all normalized by their respective total sub-domain volume to form local volume fractions:

$V_{i}^{\mathrm{loc}}=\frac{\int_{D_{i}} \rho(\phi) \mathrm{d} D}{\int_{D_{i}} \mathrm{~d} D}$

where $D_{i}$ defines a sub-domain of $D$. This makes all volume constraints, local and global, directly comparable and therefore their sensitivities as well. Hence no further normalization is necessary for the constraints.

This research uses two separate update procedures for the Lagrangian multipliers: method one for the optimization case where only the global volume inequality constraint is active and method two when the local constraints (both equality and inequality) become active.

Method One, Secant Method The first multiplier update method uses the well-known Secant method. A small optimization is performed to find the best candidate multiplier $\lambda^{*}$ for each level set update. The implementation used in the presented research is shown in equation (34).

$\lambda^{\{k+1\}}=\lambda^{\{k\}}-\frac{\lambda^{\{k\}}-\lambda^{\{k-1\}}}{h^{\{k\}}(\phi)-h^{\{k\}}(\phi)} h^{\{k\}}(\phi)$

for $k=2, \ldots, N$ where $\lambda^{\{k+1\}}$ is the multiplier suggestion for $\lambda^{*}$ at the $k$-th iteration within the Secant based optimization. The function $h^{\{k\}}(\phi)$ is defined as follows:

$h^{\{k\}}(\phi)=V^{\{k\}}(\phi)-V_{\max }^{\{k\}}$

where $V^{\{k\}}(\phi)$ is the current material volume ratio in the design domain and $V_{\max }^{\{k\}}$ is the maximum allowable volume ratio at iteration $k$. The value for $V_{\max }^{\{k\}}$ is slowly decreased from 
the initial volume ratio $V_{\text {ini }}$ to the set maximum of $V_{\max }$. This steady decrease is required for stable convergence. Without it, the material volume would reduce too rapidly, not allowing for an optimal distribution driven by the compliance based sensitivity. This method of steady decrease is a type of constraint relaxation and its form is based on the findings by Otomori et al. [25]. The slope of the relaxation is set by the parameter $N_{\mathrm{vol}}$, see equation (36).

$V_{\max }^{\{k\}}=V_{\max }+\left(V_{\mathrm{ini}}-V_{\max }\right) \max \left[0,1-\frac{k}{N_{\mathrm{vol}}}\right]$

The advantage of this Secant based update method is that the constraint value is met almost perfectly for every iteration. The disadvantage is that the current volume has to be evaluated for every iteration $k$. An optimum is reached when $\left|h^{\{k\}}(\phi)\right|<1 \cdot 10^{-4}$ or when the maximum of 25 iterations is reached. The method is initialized with: $\lambda\{1\}=10$ and $\lambda^{\{2\}}=0$ for the first LSM iteration. In subsequent iterations the Secant based method is initialized with $\lambda^{\{1\}}=\lambda^{\text {old }}$ and $\lambda^{\{2\}}=0$, where $\lambda^{\text {old }}$ is the multiplier from the previous LSM iteration.

Method Two, Augmented Method The common augmented Lagrangian function for the equality-constrained problem is defined as [5]:

$\mathscr{L}(\mathbf{x}, h(\mathbf{x}), r)=f(\mathbf{x})+\sum_{i=1}^{N}\left[\lambda_{i} h_{i}(\mathbf{x})+\frac{1}{2} r h_{i}^{2}(\mathbf{x})\right]$

where $h(\mathbf{x})$ is the equality constraint, $N$ the number of equality constraints, $\lambda$ the Lagrange multiplier for the $i$-th equality constraint and $r$ the penalty parameter. Remember that the inequality constraint problem is actually an equality constraint problem in the case of compliance minimization with a single global volume constraint. Based on the general description from equation (37), the Lagrangian from equation (29) is rewritten as follows [21,8]:

$\mathscr{L}(\phi(\mathbf{x}))=c(\phi(\mathbf{x}))+\sum_{i=0}^{N_{\mathrm{dom}}}\left[\Lambda_{i} H_{i}(\phi(\mathbf{x}))+\frac{1}{2 \tau^{\{k\}}}\left(H_{i}(\mathbf{x})\right)^{2}\right]$

The Lagrange multiplier is updated as follows:

$$
\begin{aligned}
& \lambda_{i}^{\{k+1\}}=\Lambda_{i}^{\{k\}}+\frac{1}{\tau^{\{k\}}} H_{i}(\mathbf{x}) \\
& \tau^{\{k+1\}}=\max \left(\alpha \tau^{\{k\}}, \tau_{\min }\right)
\end{aligned}
$$

where $\Lambda_{i}$ changes for $i$ as follows:

$$
\begin{array}{lll}
i=0 \rightarrow \Lambda_{i}=\lambda^{\text {glob }} & \\
i>0 \rightarrow \Lambda_{i}=\lambda_{i}^{\text {loc }} & \text { if } & D_{i} \in D_{\text {loc, eq }} \\
i>0 \rightarrow \Lambda_{i}=\max \left[\lambda_{i}^{\text {loc }}, \lambda^{\text {glob }}\right] & \text { if } & D_{i} \in D_{\text {loc, ineq }}
\end{array}
$$

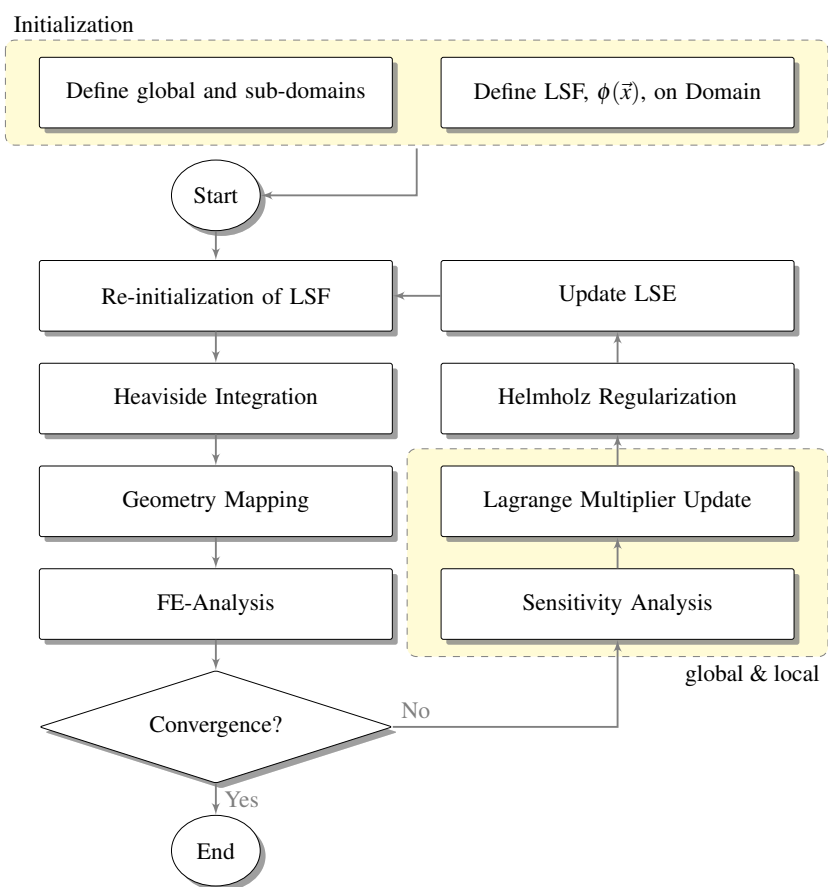

Fig. 2 Optimization Flowchart.

and where $H_{i}$ changes for $i$ as follows:

$i=0 \rightarrow H_{i}=h^{\text {glob }}$

$i>0 \rightarrow H_{i}=h_{i} \quad$ if $\quad D_{i} \in D_{\text {loc, eq }}$

$i>0 \rightarrow H_{i}=g_{i} \quad$ if $\quad D_{i} \in D_{\text {loc, ineq }}$

Parameter $\alpha=0.9$ and $\tau_{\min }=0.1$. Initial values for the Lagrange multiplier and penalty are necessary for each optimization problem. Good initial guesses are: $\lambda^{\{1\}}=4$. and $\tau^{\{1\}}=100$. An overview of the complete optimization procedure is shown in the flowchart in Figure 2.

\section{Examples}

In this section, two example problems are solved to validate the presented methods. The mechanical simulation is performed with CALCULIX [11], an open source FEM program. The material used is a quasi-isotropic material with normalized values for $E_{1}=1.542, E_{2}=1.542, G_{12}=0.206$ and $v_{12}=0.12$. Convergence is reached when the compliance does not change more than $0.1 \%$ in five subsequent iterations and the relative constraint failure is below $0.05 \%$.

\subsection{Example One}

In this example a classical Mitchell structure is solved. The general problem description is depicted in Figures 3 and 4. The design domain dimensions are given as the ratio 


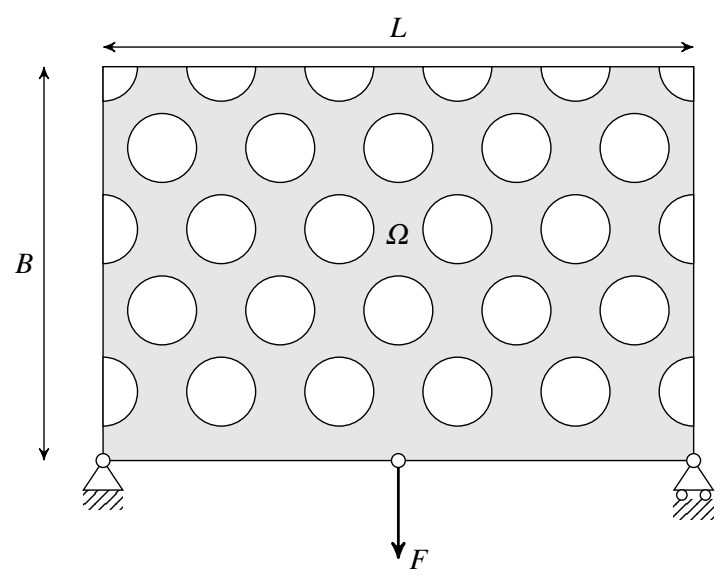

Fig. 3 Description of the Mitchell structure problem and initial boundaries.

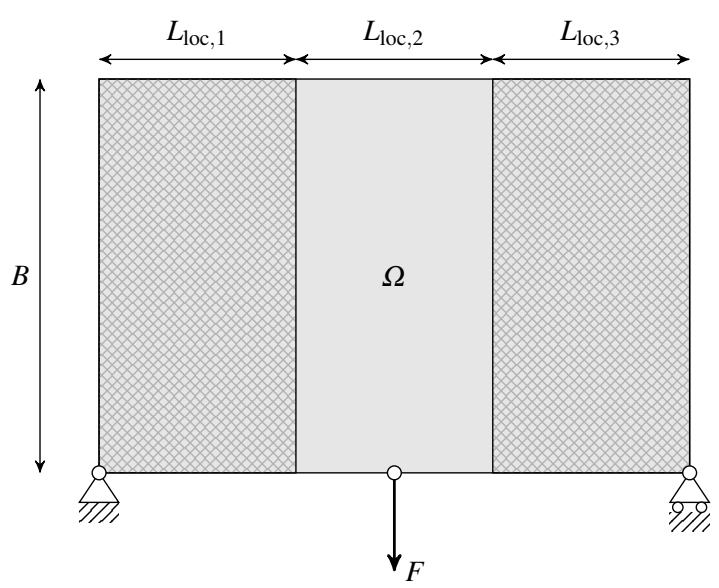

Fig. 4 Description of the local constraint domains on the Mitchell structure problem.

$L / B=1.515$. The mesh for the mechanical simulation consists of $100 \times 66$ quadrilateral 8 degree of freedom shell elements. The width of the local volume domains are specified as: $L_{\mathrm{loc}, 1}=0.35 L, L_{\mathrm{loc}, 2}=0.3 L$ and $L_{\mathrm{loc}, 3}=0.35 L$. The optimization parameters are as follows: $\beta=0 ., \tilde{R}=1.2$ and $t_{\text {fac }}=0.9$. The bandwidth parameter $h$ is set to 0.5 times the element side length. For the Lagrange multiplier update, the parameters are as follows: case one (secant method), $N_{\mathrm{vol}}=40$, case two and three (augmented method), $\tau_{0}=100$ and $\lambda_{0}=3$. The results are shown in Figure 6. The Design domain, mesh, load case and boundary conditions are the same for all three results. The global volume constraint, $V_{\text {glob }}^{\max }$, equals 0.3 for all three cases. In the first case no local constraints are active. The second case has single local inequality constraint, $V_{1,3}^{\max }=0.2$, for the combined subdomains $L_{\mathrm{loc}, 1}$ and $L_{\mathrm{loc}, 3}$, the center domain $L_{\mathrm{loc}, 2}$ is inactive. The third case has an inequality constraint, $V_{2}^{\max }=0.2$, on the sub-domain $L_{\mathrm{loc}, 2}$, the other two sub-domains are inactive. The cases are summarized in Table 1. The results show clear differences in optimal topology. The normalized opti-
Table 1 Overview of example cases and normalized optimal compliance values, $P_{\text {norm }}$, for the Michell structure problem.

\begin{tabular}{lllll}
\hline & $V_{\text {glob }}^{\max }$ & $V_{1,3}^{\max }$ & $V_{2}^{\max }$ & $P_{\text {norm }}$ \\
\hline Case 1 & 0.3 & - & - & 0.82 \\
Case 2 & 0.3 & 0.2 & - & 0.9 \\
Case 3 & 0.3 & - & 0.2 & 0.96 \\
\hline
\end{tabular}

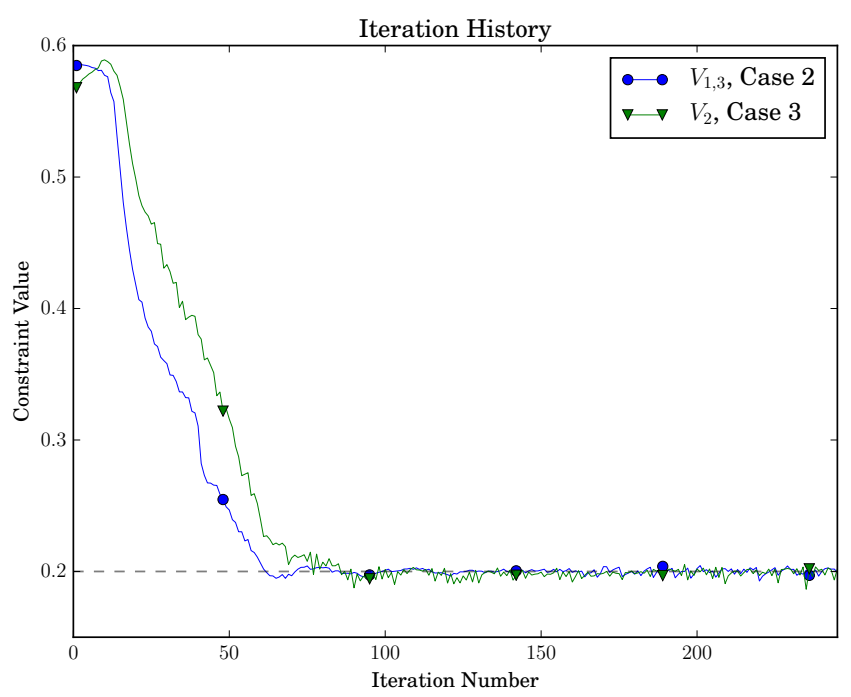

Fig. 5 Iteration history of the local volume constraints for case 2 and 3.

mal compliance values are presented in Table 1 . The result of case one is a typical, well-known, optimal topology for the Mitchell structure. The normalized minimal compliance is 0.82 . Case two shows the decrease in material volume in both outer local sub-domains, resulting in an increase of material in the central domain, because of the global volume requirement of 0.3 . The normalized minimal compliance is 0.90 , which is higher than in case one. This is to be expected as the same amount of material is distributed less optimal due to the introduced local constraints. The third case generated a different topology compared to case one and two to compensate for the lower allowed volume in the central subdomain. The normalized minimal compliance is 0.96 , which is higher than in both other cases. However, this is a logical consequence of the applied load case in combination with less material in the central sub-domain, due to the applied constraints. The optimization histories for these three cases are shown in Figure 7 and an overview of intermediate optimization results are shown in Figure 8. It should be noted that for the optimization with local constraints active, the convergence criteria were not met. Instead the optimization was halted. However, the optimization history shows reasonably stable behavior for the last 30 iterations with relative small oscillations, which here is considered as converged behavior. Similar histories can be seen for the local volume constraints, see figure 5 . 


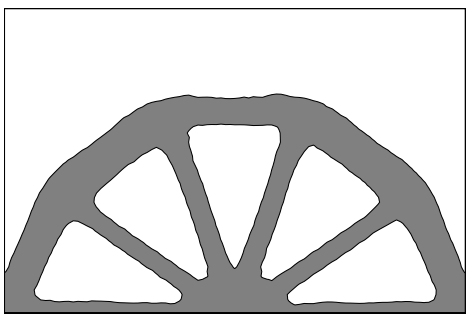

(a) Case 1: Optimum Mitchell structure with only a global constraint, $V_{\text {glob }}^{\max }=$ 0.3

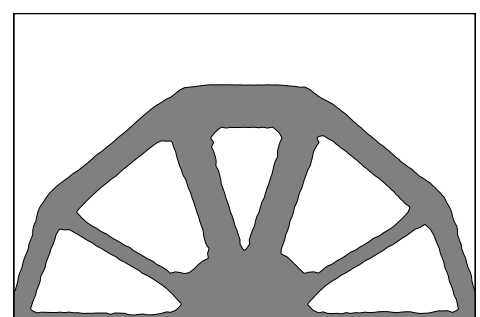

(b) Case 2: Optimum Mitchell structure with local inequality constraints, $V_{\mathrm{N}}^{\max }=0.2$, for $N=1,3$

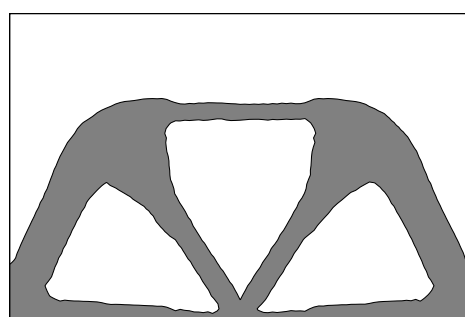

(c) Case 3: Optimum Mitchell structure with local inequality constraints, $V_{\mathrm{N}}^{\max }=0.2$, for $N=2$

Fig. 6 LSM based optima of the Mitchell structure for both without and with local volume constraints.

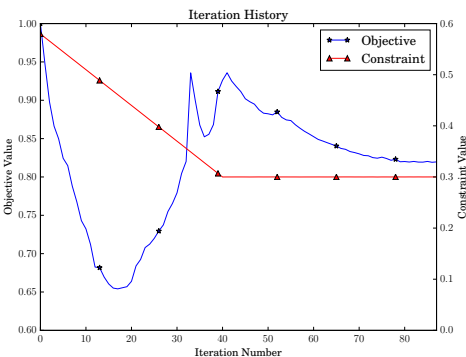

(a) Case 1

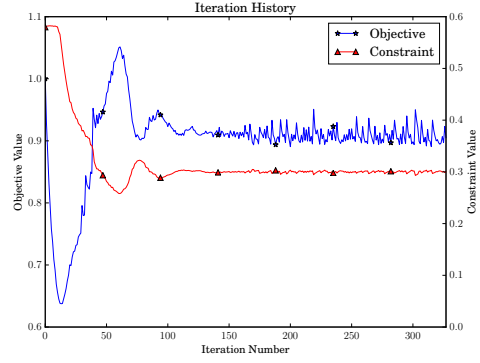

(b) Case 2

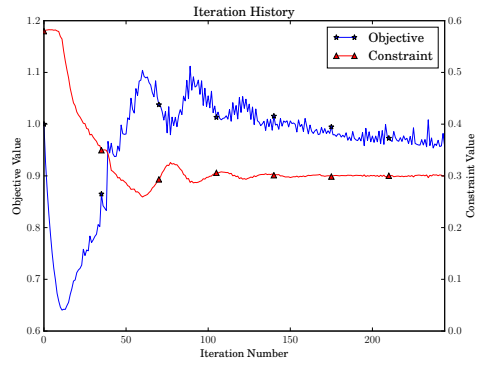

(c) Case 3

Fig. 7 Optimization histories for the cases shown in Figure 6. The objective value is normalized with respect to the initial design.

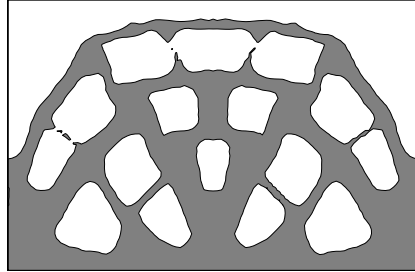

(a) Iteration 20 .

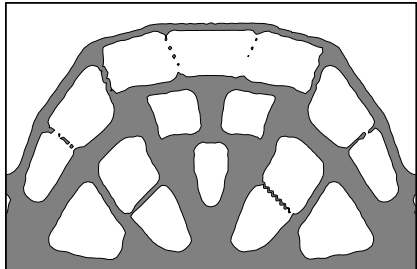

(e) Iteration 30 .

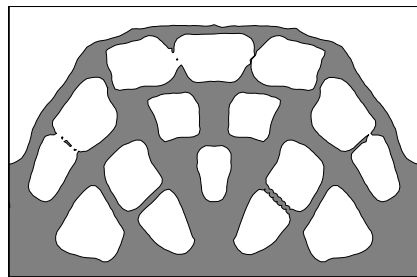

(i) Iteration 20.

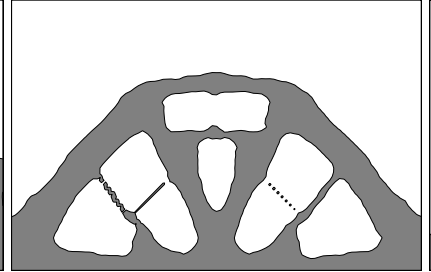

(b) Iteration 40.

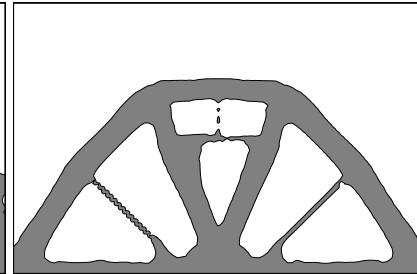

(f) Iteration 60 .

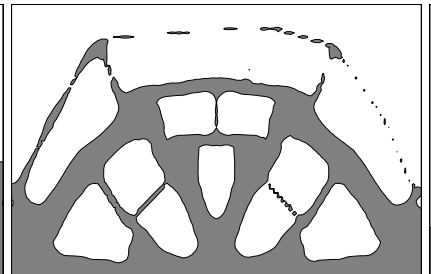

(j) Iteration 40.

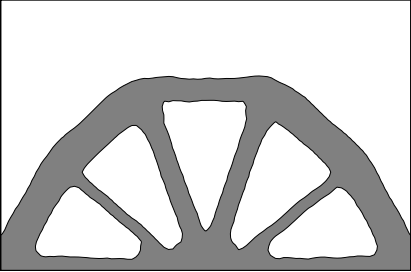

(c) Iteration 60

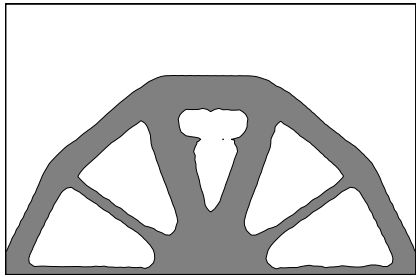

(g) Iteration 90.

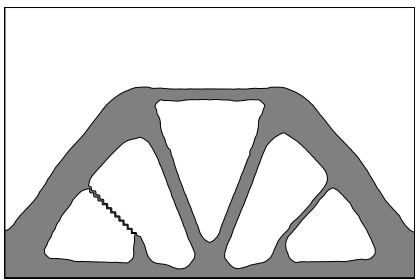

(k) Iteration 60

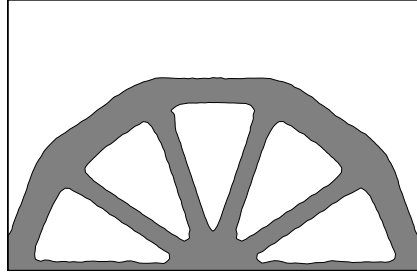

(d) Iteration 80.

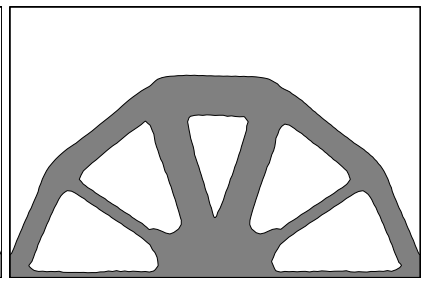

(h) Iteration 120 .

Fig. 8 LSM iteration history for the Mitchell design problem; case 1 (a to d), case 2 (e to h) and case 3 (i to l). 


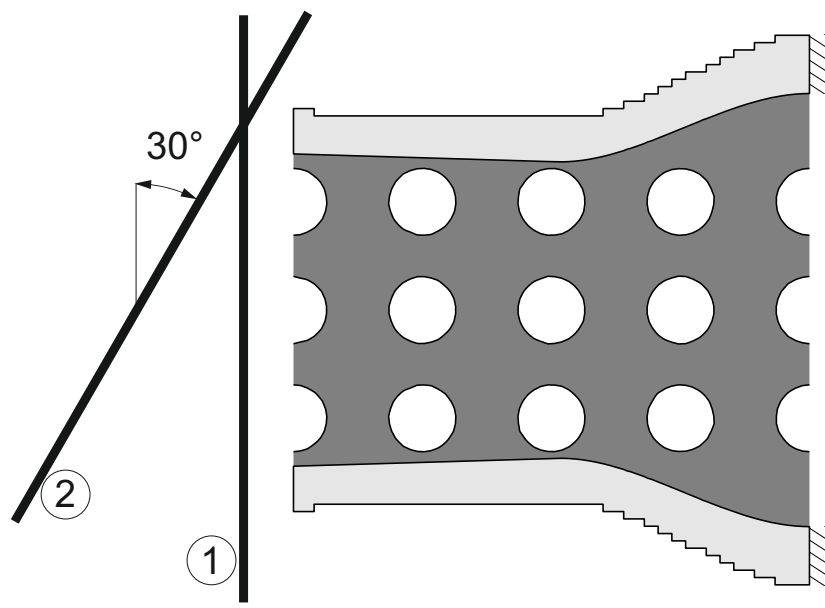

Fig. 9 Overview of the crash scenarios on the simplified vehicle front; (1) is the full width impact, (2) is the slanted impact. The structure in between the beams is initialized as a plate with holes.

The history for the reference case without local constraints active, Figure 7(a), shows a different behavior for the constraint value than the other two. This is because of the Lagrangian multiplier update scheme, which is different than for the cases with local constraints, as explained in Section 4. It can be seen however that the applied update method works as expected, showing a pre-set slope to the constraint value of 0.3 in 40 iterations (parameter $N_{\mathrm{vol}}$ ). During this time the objective value increases as expected and steadily optimizes towards convergence at constant volume.

\subsection{Example Two}

This example represents a significantly simplified vehicle front, with two CFRP-composite longitudinal beams, see Figure 9. The functions of the beams, among others, are to carry the engine and bumper and absorb some of the impact energy during a front crash. The CFRP-composite structure in between the beams is optimized for minimal compliance to find the optimal load paths and stiffen the total structure. The load cases are derived from two different crash scenarios: a full width frontal impact with a rigid barrier and an impact with a $30^{\circ}$ slanted rigid barrier see Figure 9. These impacts are represented by three different load cases, one for the full width and two for the $30^{\circ}$ barrier. The latter is divided in two separate load cases, because the impact can occur on both sides of the car. The load cases are shown in figures 11(a), 11(b) and 11(c). The manner in which these load cases were derived is not of interest for this paper and is therefore not discussed further.

It is assumed that the crush force of the structure is directly calculatable from the crush-stress of the used laminate material and the structure geometry. It is therefore also assumed that the crush force of the structure is linear propor-

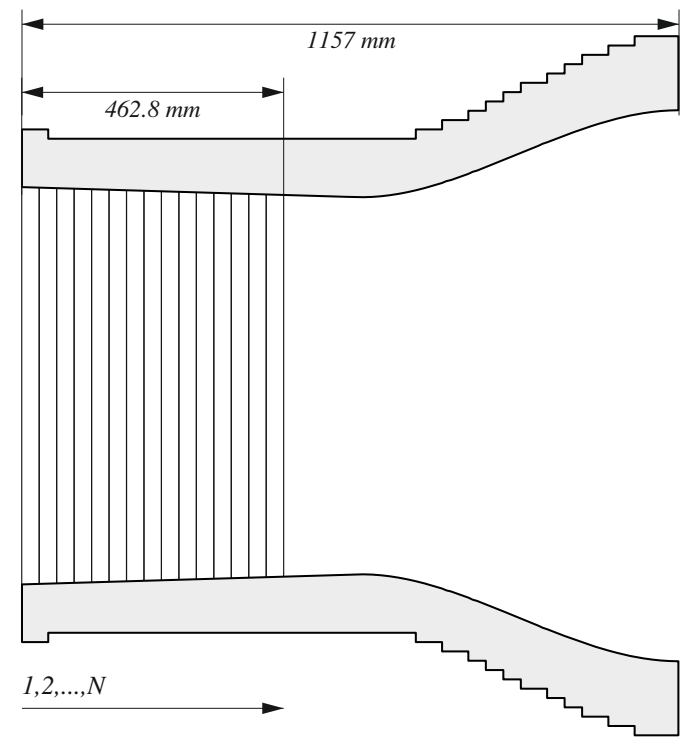

Fig. 10 Placement of the local volume domains. The columns forming the local volume domains are denoted as COL1 to COL15.

tional to the material volume encountered by the impacted surface in the impact/crush direction. For a more in-depth explanation the reader is refered to [16]. One of the primary requirements of a crashworthy composite structures, is to assure stiffness during impact, i.e. the impacted zone needs to be supported such the crushing is progressive. This supporting part ensures a stable platform for the impacted zone to show stable progressive crushing of the composite material. The minimization of the compliance increases the overall stiffness of this structure for a given material volume ratio. The control over material volume in the local domains enables a partial control over the force and therefore the dynamic crash pulse. Combining the above enables the presented method to incorporate the crash performance for composite structures in the optimization method. In short, the local volume constraints are an important link to the dynamic performance from an automotive engineering viewpoint. The crush force of the structure has a significant influence on the deceleration of the vehicle, also called the crash pulse. Large peaks in this pulse increase the likelihood of injury, therefore the optimal pulse is a square one; absorbing the maximum amount of impact energy in the available deformation space. This example shows how the local constraints are used to enforce a constant material volume in the front part of the design domain in the direction of the first impact load case; full width crash in the $\mathrm{x}$-direction. Only the front part of the design domain should show this volume continuity, as only this part of the structure is expected to be crushed in a crash event.

The front part of the design domain is divided in 15 local rectangular domains with a width of two elements, see Figure 10. By dividing the design domain in slender columns and setting subsequent constraints, the volume in perpen- 


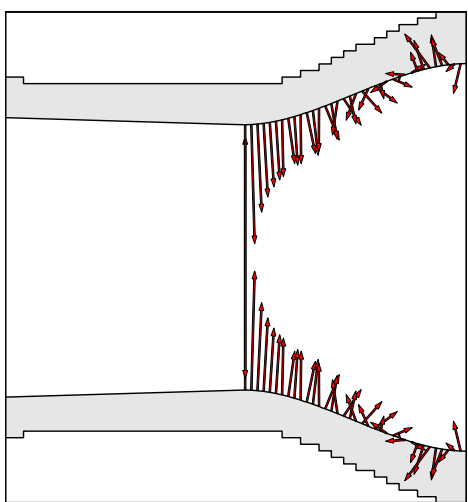

(a) Load case belonging to full width frontal impact.

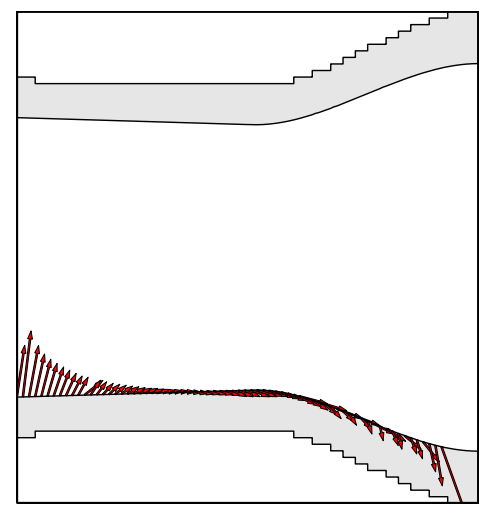

(b) Load case belonging to $30^{\circ}$ slanted barrier left-side impact.

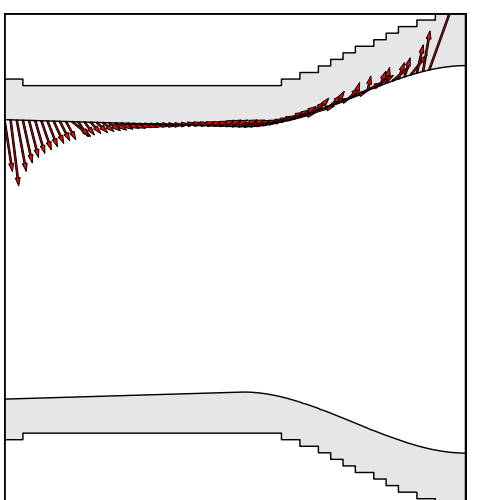

(c) Load case belonging to $30^{\circ}$ slanted barrier right-side impact.

Fig. 11 Load cases derived from two crash scenarios; one full width frontal impact and two $30^{\circ}$ slanted impacts (left and right side of vehicle). The two beams are clamped at the right end side.

dicular direction to these columns can be compelled to a predefined distribution. The mesh for the mechanical simulation consists of approximately 6000 eight degree of freedom shell elements. The right edges of the beams are fully clamped. The initial level set boundaries are distributed as a pattern of circular holes, as shown in Figure 9. The columns forming the local volume domains are denoted as COL1 to COL15. The domains COL1 to COL12 are each given a local maximum volume constraint of $V_{N}^{\max }=0.2$, COL13 has $V_{13}^{\max }=0.3, V_{14}^{\max }=0.4$ and $V_{15}^{\max }=0.5$. The global maximum volume constraint, $V_{\text {glob }}^{\max }=0.2$. The optimization parameters are as follows: $\beta=0 ., \tilde{R}=1.3$ and $t_{\mathrm{fac}}=0.9$. For the Lagrange multiplier update, the parameters are as follows: $\tau_{0}=100$ and $\lambda_{0}=7$. The bandwidth parameter $h$ is set to 0.8 times the critical element side length. The optimization result is shown in Figure 13(a) and the iteration history of the objective and global volume constraint are shown in Figure 13(b). The iteration history of the local volume constraints is shown in Figure 12. The local volume history shows that all constraints are met. The local volumes can be lower as their set maximum, because inequality constraints are used. This effect is seen in the first three columns. However, the compliance minimization drives most local volumes towards their maximum. Therefore columns 4 to 12 show a convergence to 0.2 . This result gives the required constant volume distribution in $\mathrm{x}$-direction.

To exemplify the effect of the local constraints the optimization is rerun with only the global volume constraint active, $V_{g l o b}^{\max }=0.2$. The result is shown in Figure 14(a). The resulting optimal shape looks similar to the optimum with local constraints active. However, looking at the volume ratios in the local domains in Figure 14(b), the differences becomes clear. With only the global constraint active, the volumes in most domains are significantly higher than 0.2. More importantly, the local volume ratios change notably over the domains, resulting in a discontinuous cross-

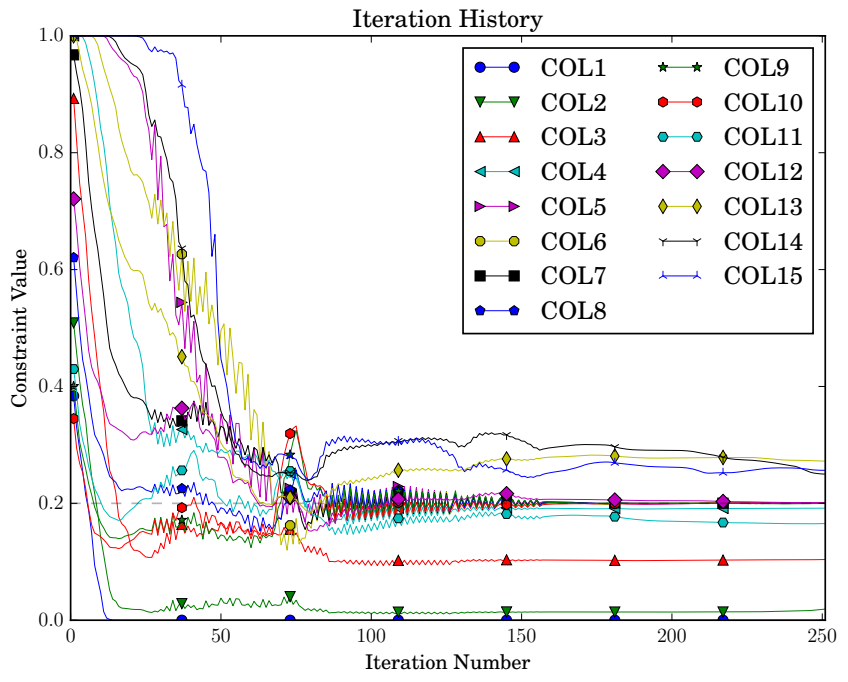

Fig. 12 Iteration history of all local volume constraints.

sectional impact area. The volume ratios with local constraints active show a largely constant value of 0.2 , going upwards in domains 13,14 and 15. This is a result of the higher constraint value in those domains, as described earlier.

\section{Discussion}

Oscillatory behavior In some cases oscillatory behavior around the optimum could be seen, on both the objective and constraint values. Especially in the first example, with the Mitchel bridge, this issue was present. It was expected that the introduction of extra Lagrange Multipliers would increase instability in the convergence history. Two possible solutions might mitigate this issue: 


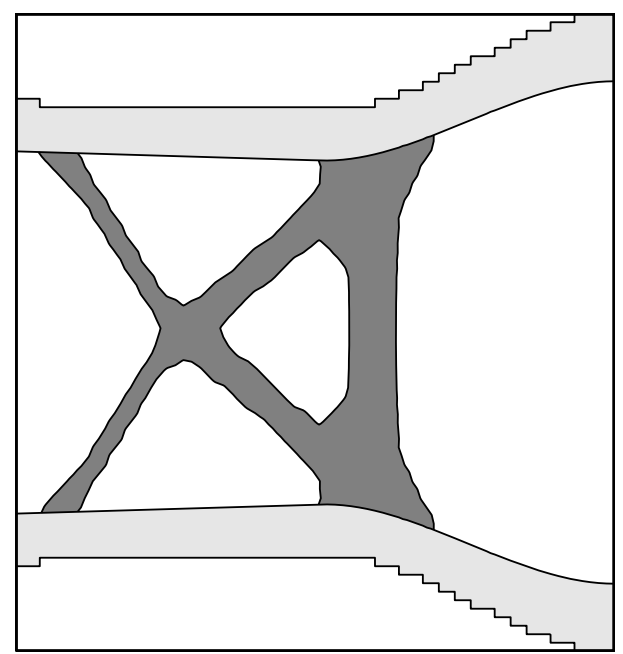

(a) Optimum of example two with active global \& local volume constraints.

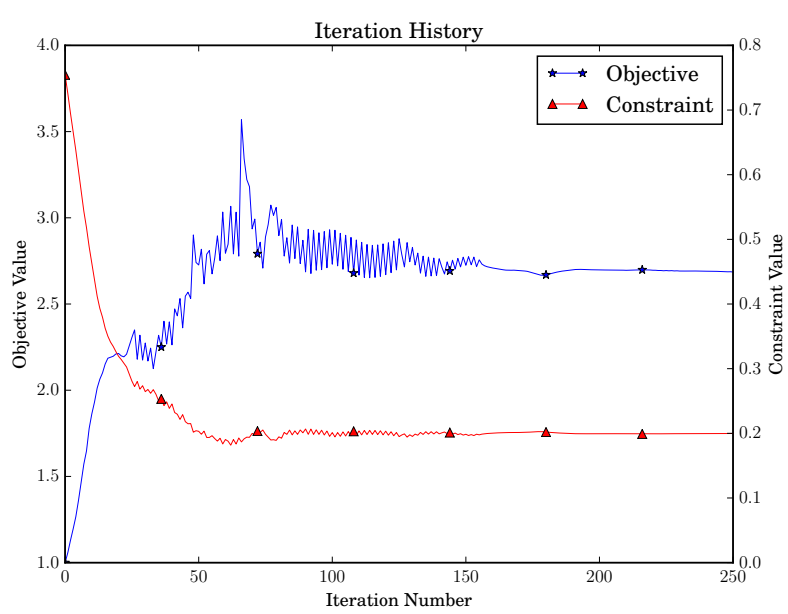

(b) Optimization history.

Fig. 13 Optimum of the automotive problem and the optimization history showing the normalized compliance and global volume.

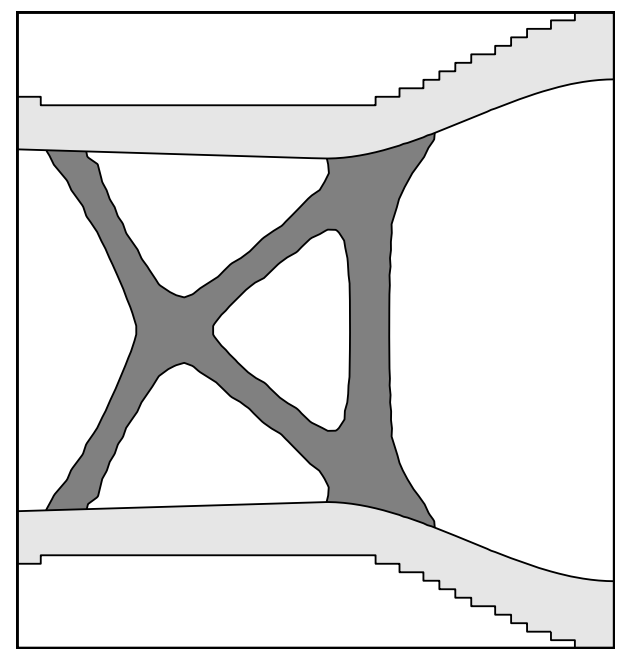

(a) Optimum of example two with only global volume constraint.

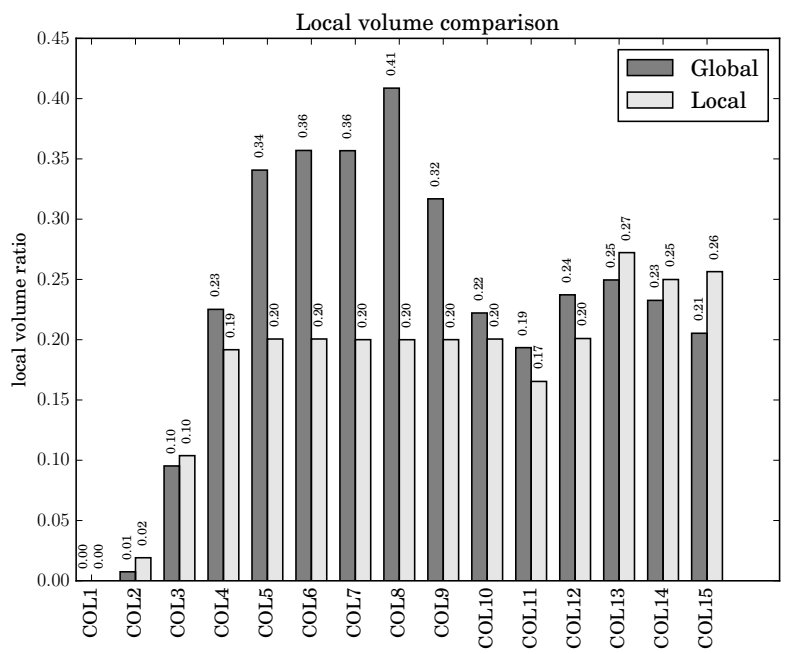

(b) Comparison of the local volumes ratios without and with local constraints.

Fig. 14 Optimum of example two with only a Global volume constraint and the comparison of the final local volumes without and with local constraints active.

- A method to decrease the time step $\Delta t$ in the LSM update procedure as soon as Oscillatory behavior is detected. This method was used in example two.

- Development and use of better Lagrange Multiplier Update Methods. This paper provides a first look into the use of sub-domain specific volume constraints. The presented multiplier update methods work well for this purpose. However, the authors realize that better update algorithms are available and future research on this topic should reflect that.

Problem Initialization The distribution and size of the holes for the problem initialization has a significant effect on the final optimized topology. Indeed, any kind of initial geome- try / shape description will show its influence on the final results. The introduction of local volume constraints increases the sensitivity to problem initialization. The dependence of the problem's initial design on the shape / topology optimization result is a well known issue within this field of research. It can be expected that the introduction of extra constraints increases this dependence. The research presented here did not focus on this issue and its effects, which are left for future developments.

Mesh Size The mesh used for both validation examples is the same and consists of relatively large elements. It is expected that a smaller mesh size will improve the results con- 
siderably, removing many of the artifacts shown in the topology results.

\section{Conclusion}

We presented a level-set based optimization framework, which can handle multiple, arbitrarily placed, volume constraints. The volume constraints were applied to nonoverlapping sub-domains of the design domain. Both equality and inequality constraint types for the volume fraction of these sub-domains can be used. To validate the method, two example design problems were presented. Both examples were solved for different configurations of volume constraints and showed promising results, presenting clear differences in topology when local volume constraints were applied. The constraints were met accurately, although in some cases some oscillatory behavior was present. Furthermore, the development of optimal geometries, including complex topology changes, was not hindered by the introduction of multiple constraints. The presented method gives the engineer increased control over the optimization problem compared to more conventional shape and topology optimization methods.

Acknowledgements The authors would like to express their gratitude to BMW Group to carry out and publish this research.

\section{A Derivatives}

The Lagrangian $\mathscr{L}$ is derived in order to transform the inequality constrained optimization problem into an unconstrained problem:

$\mathscr{L}(\phi(\mathbf{x}))=c(\phi(\mathbf{x}))+\lambda\left(g(\phi(\mathbf{x}))+s^{2}\right)$

where $s$ is a slack variable which converts the inequality into an equality constraint. The optimum is defined by meeting the KKT optimality conditions.

Let us take the compliance equal to the total strain energy:

$c=\mathbf{u}^{T} \cdot \tilde{\mathbf{K}} \cdot \mathbf{u}$

The stiffness matrix $\tilde{\mathbf{K}}(\rho)$ is determined as follows:

$\tilde{\mathbf{K}}(\rho)=\bigcup_{e=1}^{N_{e}} \rho_{e}(\phi) \mathbf{K}_{e}$

where $U$ denotes the assembly of element components, $N_{e}$ is the total number of elements, $\mathbf{K}_{e}$ is the element stiffness matrix and $\rho_{e}$ is the element density determined by the LSF values. The strain energy density can be determined as follows:

$$
\begin{aligned}
c & =\sum_{e=1}^{N_{e}} \rho_{e}(\phi) \mathbf{u}_{e}^{T} \cdot \mathbf{K}_{e} \cdot \mathbf{u}_{e} \\
& =\sum_{e=1}^{N_{e}} \tilde{H}(\phi(\mathbf{x})) \mathbf{u}_{e}^{T} \cdot \mathbf{K}_{e} \cdot \mathbf{u}_{e}
\end{aligned}
$$

The shape derivative of the Lagrangian $\mathscr{L}$ is derived as the Fréchet derivative with respect to $\phi$ as follows:

$\frac{\mathrm{d} \mathscr{L}}{\mathrm{d} \Omega}=\left\langle\frac{\mathrm{d} \mathscr{L}}{\mathrm{d} \phi}, \psi\right\rangle$

where $\psi$ is the variation of the level set function such that $\psi \in \Psi$. Combining equations (42) and (46), we get:

$\frac{\mathrm{d} \mathscr{L}}{\mathrm{d} \Omega}=\frac{\mathrm{d} c}{\mathrm{~d} \Omega}+\lambda \frac{\mathrm{d} g}{\mathrm{~d} \Omega}$

\section{A.1 Shape Derivative of the Compliance}

Now let us define the shape derivative of the strain energy density as the Fréchet derivative with respect to $\phi$ :

$\frac{\mathrm{d} c}{\mathrm{~d} \Omega}=\left\langle\frac{\mathrm{d} c}{\mathrm{~d} \phi}, \psi\right\rangle$

Now by rule of total derivative, equation (48) can be rewritten as follows:

$$
\begin{aligned}
\left\langle\frac{\mathrm{d} c}{\mathrm{~d} \phi}, \psi\right\rangle & =\left\langle\frac{\partial c}{\partial \phi}, \psi\right\rangle+\left\langle\frac{\partial c}{\partial \mathbf{u}} \frac{\mathrm{d} \mathbf{u}}{\mathrm{d} \phi}, \psi\right\rangle \\
& =\left\langle\frac{\partial c}{\partial \phi}, \psi\right\rangle+\left\langle\frac{\partial c}{\partial \mathbf{u}}, \mathbf{w}\right\rangle
\end{aligned}
$$

The partial derivatives from equation (49) are derived as follows:

$$
\begin{aligned}
& \frac{\partial c}{\partial \phi}=\sum_{e=1}^{N_{e}} \tilde{\delta}(\phi(\mathbf{x})) \mathbf{u}_{e}^{T} \cdot \mathbf{K}_{e} \cdot \mathbf{u}_{e} \\
& \frac{\partial c}{\partial \mathbf{u}}=\sum_{e=1}^{N_{e}} \tilde{H}(\phi(\mathbf{x})) 2 \mathbf{K}_{e} \cdot \mathbf{u}_{e}
\end{aligned}
$$

The Fréchet derivatives for the condition $\tilde{\mathbf{K}} \mathbf{u}-\mathbf{f}^{\mathrm{ext}}=0$ are as follows:

$\frac{\mathrm{d} \mathbf{f}^{\mathrm{ext}}}{\mathrm{d} \Omega}=0$

$\frac{\mathrm{d} \mathbf{a}}{\mathrm{d} \Omega}=\left\langle\frac{\mathrm{d} \mathbf{a}}{\mathrm{d} \phi}, \psi\right\rangle=\left\langle\frac{\partial \mathbf{a}}{\partial \phi}, \psi\right\rangle+\left\langle\frac{\partial \mathbf{a}}{\partial \mathbf{u}}, \mathbf{w}\right\rangle$

where $\mathbf{a}=\tilde{\mathbf{K}} \mathbf{u}$. The partial derivatives from equation (51) are derived as follows:

$$
\begin{aligned}
& \frac{\partial \mathbf{a}}{\partial \phi}=\sum_{e=1}^{N_{e}} \tilde{\boldsymbol{\delta}}(\phi(\mathbf{x})) \mathbf{K}_{e} \cdot \mathbf{u}_{e} \\
& \frac{\partial \mathbf{a}}{\partial \mathbf{u}}=\sum_{e=1}^{N_{e}} \tilde{H}(\phi(\mathbf{x})) \mathbf{K}_{e}
\end{aligned}
$$


From combining equations (51) and (52) follows:

$$
\begin{gathered}
\left\langle\frac{\partial \mathbf{a}}{\partial \mathbf{u}}, \mathbf{w}\right\rangle=-\left\langle\frac{\partial \mathbf{a}}{\partial \phi}, \psi\right\rangle \\
\sum_{e=1}^{N_{e}} \tilde{H}(\phi(\mathbf{x})) \mathbf{K}_{e} \cdot \mathbf{w}=\ldots \\
-\sum_{e=1}^{N_{e}} \tilde{\delta}(\phi(\mathbf{x})) \mathbf{K}_{e} \cdot \mathbf{u}_{e} \psi
\end{gathered}
$$

Now combining equations (49) and (50) and substituting equation (53), we get the following:

$$
\begin{array}{r}
\frac{\mathrm{d} c}{\mathrm{~d} \Omega}=\sum_{e=1}^{N_{e}} \tilde{\boldsymbol{\delta}}(\phi(\mathbf{x})) \mathbf{u}_{e}^{T} \cdot \mathbf{K}_{e} \cdot \mathbf{u}_{e} \psi+\ldots \\
\sum_{e=1}^{N_{e}} \tilde{H}(\phi(\mathbf{x})) 2 \mathbf{K}_{e} \cdot \mathbf{u}_{e} \cdot \mathbf{w} \\
=\sum_{e=1}^{N_{e}} \tilde{\delta}(\phi(\mathbf{x})) \mathbf{u}_{e}^{T} \cdot \mathbf{K}_{e} \cdot \mathbf{u}_{e} \psi-\ldots \\
\sum_{e=1}^{N_{e}} 2 \tilde{\boldsymbol{\delta}}(\phi(\mathbf{x})) \mathbf{u}_{e}^{T} \cdot \mathbf{K}_{e} \cdot \mathbf{u}_{e} \psi
\end{array}
$$

This leads to the following relation for the shape derivative of the strain energy density:

$$
\frac{\mathrm{d} c}{\mathrm{~d} \Omega}=-\sum_{e=1}^{N_{e}} \tilde{\delta}(\phi(\mathbf{x})) \mathbf{u}_{e}^{T} \cdot \mathbf{K}_{e} \cdot \mathbf{u}_{e} \psi
$$

\section{A.2 Shape Derivative of the Global Volume Constraint}

The derivation of the shape derivative of the Volume constraint follows the same procedure as with the compliance. The volume $V$ is expressed as follows:

$V(\phi(\mathbf{x}))=\sum_{e=1}^{N_{e}} \tilde{H}(\phi(\mathbf{x}))$

The shape derivative of the constraint $g(\phi(\mathbf{x}))$ is derived as follows:

$$
\frac{\mathrm{d} g}{\mathrm{~d} \Omega}=\left\langle\frac{\mathrm{d} g}{\mathrm{~d} \phi}, \psi\right\rangle=\left\langle\frac{\partial g}{\partial \phi}, \psi\right\rangle
$$

This leads to the following definition of the constraint shape derivative:

$$
\frac{\mathrm{d} g}{\mathrm{~d} \Omega}=\sum_{e=1}^{N_{e}} \tilde{\delta}(\phi(\mathbf{x})) \psi
$$

\section{A.3 Shape Derivative of the Lagrangian}

The shape derivative of the Lagrangian can now be defined by substituting the results from equations (55) and (58) into equation (47) and taking the derivative with respect to the LSF values:

$$
\frac{\mathrm{d} \mathscr{L}}{\mathrm{d} \phi}=\sum_{e=1}^{N_{e}} \tilde{\delta}(\phi(\mathbf{x}))\left[-\mathbf{u}_{e}^{T} \cdot \mathbf{K}_{e} \cdot \mathbf{u}_{e}+\lambda\right]
$$

The boundary normal velocity $V_{N}(\mathbf{x}, t)$ can now be defined as:

$V_{N}(\mathbf{x}, t)=\frac{\mathrm{d} \mathscr{L}}{\mathrm{d} \phi}$

The Lagrangian formulation of the optimization problem contains a slack variable $s$ to account for the inequality constraint. The switching condition from the KKT conditions can be satisfied in two ways:

$-\lambda=0$ : This implies that the inequality condition is inactive, meaning that the suggested optimum features a lower volume fraction than $V_{\max }$. However, for problems with fixed boundary conditions and fixed loads, not considering body forces, the compliance is minimized when the design domain is completely filled with material. This fact makes this case physically irrelevant.

- $s=0$ : Zero slack implies an active inequality constraint, $g(\phi)=0$, indicating that $V(\phi)=V_{\max }$ for the optimum solution.

These cases show that the optimum will always lie at $V(\phi)=$ $V_{\max }$. As a result of this, one could define the volume constraint in equation (21) as an equality constraint. The slack variable $s$ is now redundant and omitted.

\section{References}

1. Allaire, G., Gournay, F.D., Jouve, F., Toader, A.M.: Structural optimization using topological and shape sensitivity via a level set method. Control and Cybernetics 34(1), 59-80 (2005)

2. Allaire, G., Jouve, F.: Minimum stress optimal design with the level set method. Engineering Analysis with Boundary Elements 32(11), 909-918 (2008)

3. Allaire, G., Jouve, F., Toader, A.M.: A level-set method for shape optimization. Comptes Rendus Mathématique 334(12), 11251130 (2002)

4. Allaire, G., Jouve, F., Toader, A.M.: Structural optimization using sensitivity analysis and a level-set method. Journal of Computational Physics 194(1), 363-393 (2004)

5. Arora, J.: Introduction to optimum design. Academic Press (2012)

6. Bendsøe, M.P., Sigmund, O.: Material interpolation schemes in topology optimization. Archive of applied mechanics 69(9-10), 635-654 (1999)

7. Bruggi, M., Duysinx, P.: Topology optimization for minimum weight with compliance and stress constraints. Structural and Multidisciplinary Optimization 46(3), 369-384 (2012)

8. Challis, V.J.: A discrete level-set topology optimization code written in Matlab. Structural and Multidisciplinary Optimization 41(3), 453-464 (2010)

9. De Ruiter, M., Van Keulen, F.: Topology optimization: approaching the material distribution problem using a topological function description. In: International conference on engineering computational technology, pp. 111-119 (2000)

10. De Ruiter, M.J., Van Keulen, F.: Topology optimization using a topology description function. Structural and Multidisciplinary Optimization 26(6), 406-416 (2004)

11. dHondt, G., Wittig, K.: CalculiX Users Guide. MTU

12. van Dijk, N., Langelaar, M., van Keulen, F.: Explicit level-setbased topology optimization using an exact Heaviside function and consistent sensitivity analysis. International Journal for $\mathrm{Nu}-$ merical Methods in Engineering 91(1), 67-97 (2012) 
13. van Dijk, N.P., Maute, K., Langelaar, M., Van Keulen, F.: Level-set methods for structural topology optimization: A review. Structural and Multidisciplinary Optimization 48(3), 437-472 (2013)

14. Duddeck, F.: Multidisciplinary optimization of car bodies. Structural and Multidisciplinary Optimization 35(4), 375-389 (2008)

15. Duysinx, P., Bendsøe, M.P.: Topology optimization of continuum structures with local stress constraints. International journal for numerical methods in engineering 43(8), 1453-1478 (1998)

16. Hesse, S.H., Lukaszewicz, D.H.J.A., Duddeck, F.: A method to reduce design complexity of automotive composite structures with respect to crashworthiness. Composite Structures 129, 236-249 (2015)

17. James, K.a., Lee, E., Martins, J.R.R.a.: Stress-based topology optimization using an isoparametric level set method. Finite Elements in Analysis and Design 58, 20-30 (2012)

18. Kawamoto, A., Matsumori, T., Yamasaki, S., Nomura, T., Kondoh, T., Nishiwaki, S.: Heaviside projection based topology optimization by a PDE-filtered scalar function. Structural and Multidisciplinary Optimization 44(1), 19-24 (2011)

19. Kreissl, S.: Topology Optimization of Flow Problems Modeled by the Incompressible Navier-Stokes Equations. Ph.D. thesis, University of Colorado Boulder (2011)

20. Le, C., Norato, J., Bruns, T., Ha, C., Tortorelli, D.: Stress-based topology optimization for continua. Structural and Multidisciplinary Optimization 41(4), 605-620 (2010)

21. Luo, J., Luo, Z., Chen, L., Tong, L., Wang, M.Y.: A semi-implicit level set method for structural shape and topology optimization. Journal of Computational Physics 227, 5561-5581 (2008)

22. Luo, Z., Wang, M.Y., Wang, S., Wei, P.: A level set-based parameterization method for structural shape and topology optimization. International Journal for Numerical Methods in Engineering 76(1), 1-26 (2008)

23. Osher, S., Sethian, J.A.: Fronts Propagating with CurvatureDependent Speed: Algorithms Based on Hamilton-Jacobi Formulations. Journal of Computational Physics 79, 12-49 (1988)

24. Osher, S.J., Santosa, F.: Level Set Methods for Optimization Problems Involving Geometry and Constraints: I. Frequencies of a Two-Density Inhomogeneous Drum. Journal of Computational Physics 171(1), 272-288 (2001)

25. Otomori, M., Yamada, T., Izui, K., Nishiwaki, S.: Topology Optimization Based on the Level Set Method Using Mathematical Programming. Transactions of the Japan Society of Mechanical Engineers Series C 77, 4001-4014 (2011)

26. Otomori, M., Yamada, T., Izui, K., Nishiwaki, S.: Matlab code for a level set-based topology optimization method using a reaction diffusion equation. Structural and Multidisciplinary Optimization 51(5), 1159-1172 (2014)

27. Paris, J., Navarrina, F., Colominas, I., Casteleiro, M.: Topology optimization of continuum structures with local and global stress constraints. Structural and Multidisciplinary Optimization 39(4), 419-437 (2009)

28. Sethian, J., Wiegmann, A.: Structural Boundary Design via Level Set and Immersed Interface Methods. Journal of Computational Physics 163(2), 489-528 (2000)

29. Van Miegroet, L., Duysinx, P.: Stress concentration minimization of 2D filets using X-FEM and level set description. Structural and Multidisciplinary Optimization 33(4-5), 425-438 (2007)

30. Wang, M.Y., Wang, X., Guo, D.: A level set method for structural topology optimization. Computer Methods in Applied Mechanics and Engineering 192(1-2), 227-246 (2003)

31. Wang, S., Wang, M.Y.: Radial basis functions and level set method for structural topology optimization. International journal for numerical methods in engineering 65(12), 2060-2090 (2006)

32. Yamasaki, S., Nishiwaki, S., Yamada, T., Izui, K., Yoshimura, M.: A structural optimization method based on the level set method using a new geometry-based re-initialization scheme. International Journal for Numerical Methods in Engineering 83(12), 1580-1624 (2010)
33. Yamasaki, S., Nomura, T., Kawamoto, A., Sato, K., Nishiwaki, S.: A level set-based topology optimization method targeting metallic waveguide design problems. International Journal for Numerical Methods in Engineering 87(9), 844-868 (2011)

34. Yang, R.J., Chen, C.J.: Stress-based topology optimization. Structural and Multidisciplinary Optimization 12(2), 98-105 (1996)

35. Zhou, M., Rozvany, G.: The coc algorithm, part ii: Topologi$\mathrm{cal}$, geometrical and generalized shape optimization. Computer Methods in Applied Mechanics and Engineering 89(1), 309 - 336 (1991) 